\title{
Role of the electron spin in determining the coherence of the nuclear spins in a quantum dot
}

\author{
Gunter Wüst ${ }^{1}$, Mathieu Munsch ${ }^{1 \star}$, Franziska Maier${ }^{1}$, Andreas V. Kuhlmann', Arne Ludwig², \\ Andreas D. Wieck², Daniel Loss', Martino Poggio' and Richard J. Warburton'
}

\begin{abstract}
A huge effort is underway to develop semiconductor nanostructures as low-noise qubits. A key source of dephasing for an electron spin qubit in GaAs and in naturally occurring Si is the nuclear spin bath. The electron spin is coupled to each nuclear spin by the hyperfine interaction. The same interaction also couples two remote nuclear spins via a common coupling to the delocalized electron. It has been suggested that this interaction limits both electron and nuclear spin coherence, but experimental proof is lacking. We show that the nuclear spin decoherence time decreases by two orders of magnitude on occupying an empty quantum dot with a single electron, recovering to its original value for two electrons. In the case of one electron, agreement with a model calculation verifies the hypothesis of an electron-mediated nuclear spin-nuclear spin coupling. The results establish a framework to understand the main features of this complex interaction in semiconductor nanostructures.
\end{abstract}

A n electron spin qubit can be controlled electrically, implying fast and scalable operation ${ }^{1}$. A key issue is that an electron spin in GaAs or Si couples to the nuclear spins in the host material via the contact hyperfine interaction ${ }^{2}$. The first-order electron spin-nuclear spin flip-flop processes can be suppressed by applying a magnetic field, exploiting the mismatch in the electron and nuclear gyromagnetic ratios. The interaction along the magnetic field direction remains and leads to small electron spin dephasing times ${ }^{3}$ unless the nuclear spins are prepared carefully ${ }^{4,5}$. The nuclear spin dynamics are slow, such that dynamic decoupling or real-time Hamiltonian estimation schemes are successful at prolonging electron spin coherence ${ }^{5-8}$. Arguably, therefore, these first-order hyperfine effects can be dealt with. However, the second-order hyperfine effects remain and it has been proposed that they determine the ultimate limit on the electron spin coherence $^{9-11}$. This point is very important for the development of a viable spin qubit yet is difficult to prove experimentally-many factors (phonons, co-tunnelling, charge noise ${ }^{12}$ ) influence the electron spin decoherence-and the theory is complex ${ }^{13,14}$.

We attack this problem by probing the interaction from the point of view of the nuclear spins. In the absence of an electron the nuclear spins are largely isolated, interacting with each other only by the weak dipoledipole interaction, leading to long coherence times (in the millisecond regime $)^{15,16}$. This is an ideal starting point: the nuclear spin coherence is a sensitive probe of any interaction activated by the presence of a single electron. Specifically, the second-order flip-flop process should lead to a measurable effect on the nuclear spin coherence.

The nuclear spin-nuclear spin flip-flop arises via a common coupling of two remote nuclear spins to a delocalized electron that couples the nuclear spins together. The interaction has been invoked to explain an electron spin dependence of the nuclear spin diffusion rate out from a quantum dot into the surrounding material ${ }^{17}$. However, the consequences of this coupling for nuclear spin coherence on the nanoscale are largely unexplored.

Here we probe the nuclear spins associated with a single selfassembled InGaAs quantum dot. There are a few advantages of this approach. First, the large confinement energies lead to a pronounced Coulomb blockade: the quantum dot charge can be controlled precisely via the bias applied to a gate ${ }^{18}$. This allows the nuclear spin coherence to be measured both with and without an electron. Second, the nuclear spins can be both polarized (dynamic nuclear polarization; DNP) and read out optically ${ }^{19}$. DNP represents a laser cooling of the nuclear spins down to millikelvin temperatures ${ }^{20}$, creating large population differences between the nuclear spin levels. Nuclear polarization results in subtle shifts in the optical resonance frequency that can be measured very precisely. Nuclear magnetic resonance (NMR) sensitive to just $\sim 1,000$ nuclear spins has been achieved ${ }^{16,20,21}$. Finally, the large quadrupole shifts of the nuclear spin levels in this system ${ }^{20-22}$ arguably represent a simplification: the nuclei can be treated as a collection of spin-1/2 spins, an ideal test-bed for theory.

NMR on a self-assembled quantum dot with charge control The experiment measures the coherence of the nuclear spins associated with an InGaAs quantum dot for different charge states, empty (0), singly occupied (1e) and doubly occupied (2e). A static magnetic field is applied along the growth direction, $z$; an oscillating magnetic field is applied in-plane, in the $x$ direction. Specifically, we measure the coherence associated with the 'central' transition (nuclear spin $\left.I_{z}=-(1 / 2) \leftrightarrow I_{z}=+(1 / 2)\right)$ of the ${ }^{75}$ As and ${ }^{115}$ In isotopes. Owing to the in-built strain, each nucleus experiences an electric field gradient that leads to quadrupole shifts of the bare levels ${ }^{20-22}$, as shown in Fig. 1a, the eigenenergies in the laboratory frame. The in-built strain is site-dependent, resulting in a spread of electric field gradients across the quantum dot-particularly across the electron wave function. For ${ }^{75}$ As with $I=3 / 2$, the first-order quadrupole effect shifts the $I_{z}=-(3 / 2) \leftrightarrow I_{z}=-(1 / 2)$ transition to lower frequencies and the $I_{z}=+(1 / 2) \leftrightarrow I_{z}=+(3 / 2)$ transition to higher frequencies, but the frequency of the central transition is shifted only in secondorder. The NMR spectrum consists of a central peak at $v_{Z}$, inhomogeneously broadened by the second-order quadrupole effects, and well-separated sidebands at $v_{\mathrm{Z}} \pm v_{\mathrm{Q}}^{(1)}$ where $v_{\mathrm{Z}}$ is the Zeeman frequency and $v_{\mathrm{Q}}^{(1)}$ is the first-order quadrupole shift ${ }^{21}$. For ${ }^{115} \mathrm{In}$ with $I=9 / 2$ there are nine NMR transitions but, as for ${ }^{75} \mathrm{As}$, the 
a
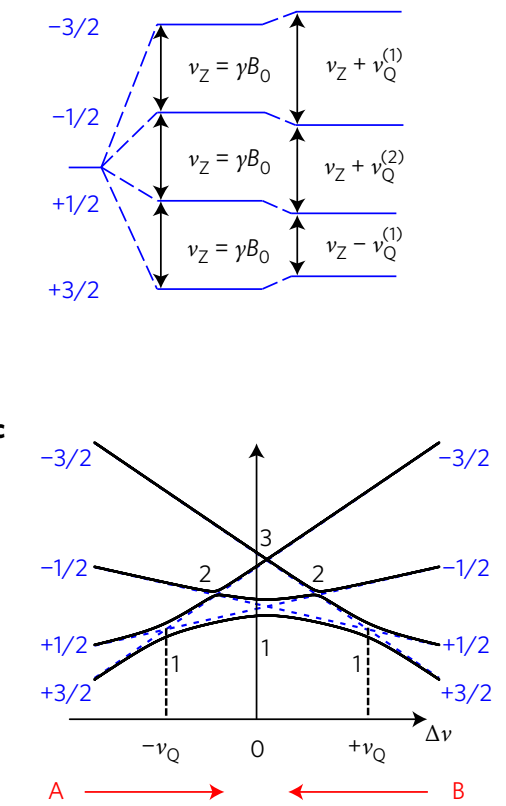
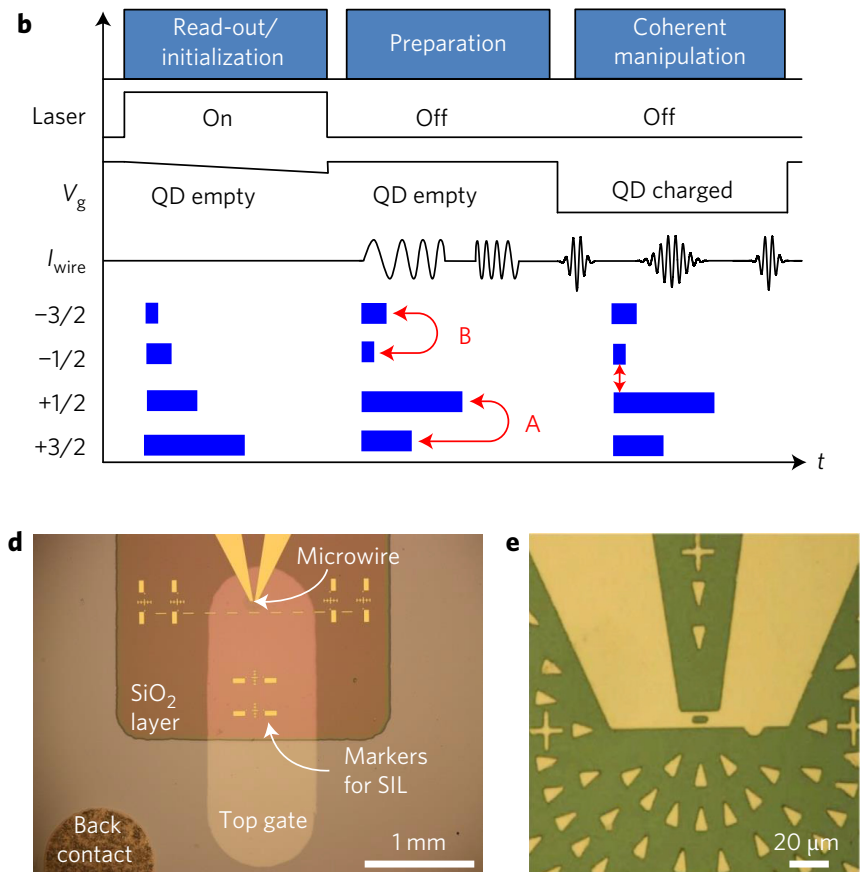

Figure 1 | Concepts. a, Energy levels for a spin $I=3 / 2$ in the laboratory frame. The static field $B_{z}$ causes the spin states to split into the Zeeman ladder with spacing Larmor frequency $\nu_{Z}$. Quadrupole effects result in alterations to the level spacing; the central transition frequency is only shifted by second-order terms. b. The measurement cycle. Read-out/initialization involves detecting the resonance fluorescence from the empty quantum dot (QD; neutral exciton) excited with a narrow-band laser: this both reads the previous nuclear spin polarization and sets a new state. In the preparation stage, two chirped pulses are applied $(A$ and $B)$ that swap the populations $+(3 / 2) \leftrightarrow+(1 / 2)$ and $-(3 / 2) \leftrightarrow-(1 / 2)$ to maximize the population difference between the $+(1 / 2)$ and $-(1 / 2)$ states. In the coherent manipulation stage a particular bias (which controls the quantum dot charge, Fig. 4) is applied to the gate of the device and then a pulse of a.c. current $\left(I_{\text {wire }}\right)$ is applied to the microwire at the radiofrequency of the central transition. Finally, the bias is reset to the starting value. c, Energy levels for a $3 / 2$ spin in the rotating frame versus radiofrequency detuning in the limit of $\nu_{\mathrm{Q}} \gg \nu_{\mathrm{RF}}$ where $\nu_{\mathrm{RF}}$ is the Rabi coupling. The preparation pulses are

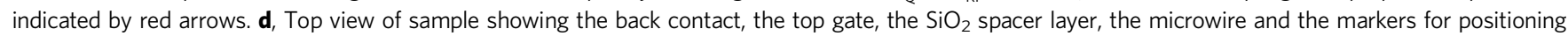
the solid immersion lens (SIL). e, Zoom-in of the microwire. A hole in the wire enables optical access to the quantum dot; the triangular markers facilitate the positioning of the sample in the microscope.

central transition is unaffected by the quadrupole interaction to first order. Hence, in the limit $v_{\mathrm{Q}}^{(1)} \gg v_{\mathrm{RF}}$ for each nuclear spin the $I_{z}=-(1 / 2) \leftrightarrow I_{z}=+(1 / 2)$ NMR transition can be thought of as a quasi-two-level system: on driving at frequency $v_{Z}$ the population is largely confined to the $I_{z}= \pm(1 / 2)$ subspace. The eigenenergies in the rotating frame are shown in Fig. 1c for a realistic quadrupole frequency $v_{\mathrm{Q}}^{(1)}(2 \mathrm{MHz})$ and Rabi coupling $v_{\mathrm{RF}}(100 \mathrm{kHz})$. The strongest avoided crossings occur when the bare states have a difference in angular momentum of one quantum unit ${ }^{20}$.

The total number of nuclear spins is $\sim 10^{5}$ (ref. 23) and the number of ${ }^{115}$ In nuclei $\sim 10^{4}$. These are very small numbers in NMR terms, demanding a very sensitive read-out. The optical resonance frequency is sensitive to the nuclear spin polarization ${ }^{2,19}$ and given the high sensitivity of optical probes, this enables the nuclear spin polarization to be measured with high precision. Even with high-resolution, high-sensitivity optical spectroscopy, the thermal differences in the nuclear level populations are too small to give useful signals even at $4 \mathrm{~K}$ in large static magnetic fields. This problem is solved by first polarizing the nuclear spins optically, creating a DNP. Second, we swap the populations of the $+(3 / 2)$ and $+(1 / 2)$ states and the populations of the $-(3 / 2)$ and $-(1 / 2)$ states, creating a non-thermal distribution with the maximum population difference between the $+(1 / 2)$ and $-(1 / 2)$ states (Fig. 1b). To do this, we use adiabatic passage at the $-(3 / 2)$, $-(1 / 2)$ avoided crossing, sweeping the NMR frequency from well below $v_{\mathrm{Z}}$ to just beyond $v_{\mathrm{Z}}-v_{\mathrm{Q}}$ (process $\mathrm{A}$ in Fig. $1 \mathrm{~b}, \mathrm{c}$ ); and adiabatic passage at the $+(3 / 2),+(1 / 2)$ avoided crossing, sweeping the NMR frequency from well above $v_{\mathrm{Z}}$ to just below $v_{\mathrm{Z}}+v_{\mathrm{Q}}$ (process B in Fig. 1b,c).
The quantum dot charge is controlled precisely by a Coulomb blockade: there are abrupt steps in the charge state, $0 \rightarrow 1 e \rightarrow 2 e$ as a function of bias $V_{\mathrm{g}}$ applied to the device ${ }^{18,24}$. The nuclear spin polarization is manipulated by a.c. magnetic fields (of a few millitesla) created by passing a.c. currents through an on-chip microwire (Fig. 1d,e). We drive the optical transition with a resonant laser, detecting the resonance fluorescence ${ }^{25,26}$. Resonant driving enables us to establish nuclear spin polarizations up to $\sim 30 \%$ in an applied magnetic field ${ }^{27}$. We achieve a sensitivity to just 1,000 nuclear spins in single-shot read-out ${ }^{20}$ and a sensitivity to 100 nuclear spins in this experiment.

\section{Rabi oscillations and Hahn echo measurements}

We present first a Rabi oscillation experiment. A pulse at the ${ }^{75} \mathrm{As}$ central transition frequency is applied to the microwire. Figure $2 \mathrm{a}$ shows the NMR signal as a function of pulse duration. A clear oscillation is observed-a Rabi oscillation-as the population is driven coherently between the $I_{z}=-(1 / 2)$ and $+(1 / 2)$ states. The period corresponds closely to the expected result, double the ${ }^{75}$ As gyromagnetic ratio (the factor of two is the effective coupling and arises on folding the system to an effective spin-1/2 system ${ }^{28}$ ). When the quantum dot is empty, the Rabi oscillations decay with a time constant of $50 \mu \mathrm{s}$. Given that the coherence time associated with this transition is in the millisecond regime ${ }^{16}$, this decay corresponds not to a loss of coherence but to an inhomogeneous broadeningthe second-order quadrupole shift to the central transition $v_{\mathrm{Q}}^{(2)}$. To determine this inhomogeneous broadening $\delta v_{\mathrm{Q}}^{(2)}$, we calculate the response of an ensemble of coherent two-level systems with a Gaussian distribution of the central frequencies. Figure 2a shows 

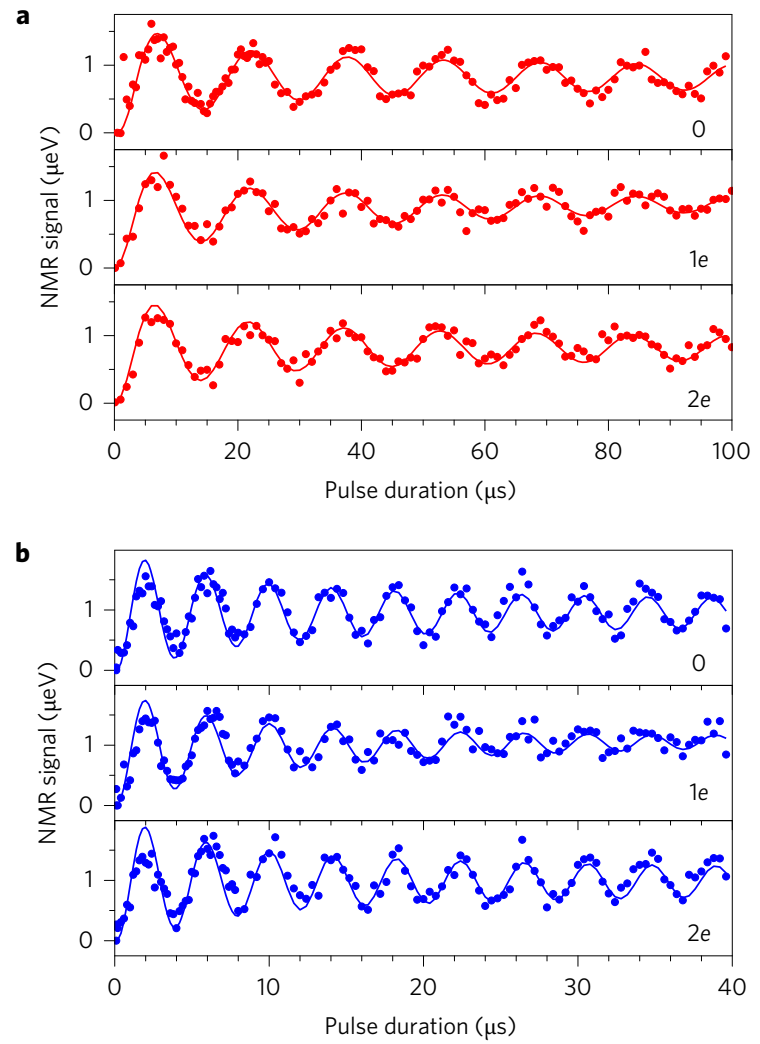

Figure 2 | Rabi oscillations of the nuclear spin ensemble. Following nuclear state preparation, we charge the quantum dot (to $0,1 e$ or 2e states) and apply a radiofrequency pulse resonant with the central nuclear transition. The bias is set to the centre of the charging plateau in the case of an occupancy of one electron. $\mathbf{a}, \mathbf{b}$, The NMR signal (the optical shift) is shown versus pulse duration for As (a) and In (b) for occupancy with zero, one electron or two electrons as indicated. The solid lines represent the calculated response of an inhomogeneous distribution (FWHM of the central frequency $\delta \nu_{\mathrm{Q}}^{(2)}$ ) of two-level emitters with a Gaussian distribution of the resonance frequency. For ${ }^{75} \mathrm{As}$ at zero occupancy, the Rabi oscillations are fitted with Rabi coupling $\nu_{\text {eff }}=64 \mathrm{kHz}$ (radiofrequency magnetic field $\left.B_{\mathrm{RF}}=4.4 \mathrm{mT}\right), \delta \nu_{\mathrm{Q}}=71.7 \mathrm{kHz}$, taking in this case a large $T_{2}\left(T_{2}=5 \mathrm{~ms}\right)$. In the 1e state, the fit is excellent with the same value of $\nu_{\text {eff }}$ and $\delta \nu_{\mathrm{Q}}^{(2)}$ but with $T_{2}=108 \mu \mathrm{s}$. For In, for occupancy zero the fit yields $\nu_{\text {eff }}=241 \mathrm{kHz}$, $\delta \nu_{\mathrm{Q}}^{(2)}=146 \mathrm{kHz}$ and $B_{\mathrm{RF}}=5.2 \mathrm{mT}$. For occupancy of one electron, the fit is excellent with the same $\nu_{\text {eff }}$ and $\delta \nu_{\mathrm{Q}}^{(2)}$ but with $T_{2}=25 \mu \mathrm{s}$. For both ${ }^{75} \mathrm{As}$ and ${ }^{115} \mathrm{In}$, the fit for $2 \mathrm{e}$ is excellent with the same parameters as for occupancy zero. The static magnetic field is $B_{z}=6.6 \mathrm{~T}$, temperature $4.2 \mathrm{~K}$.

an excellent fit to the data with $\delta v_{\mathrm{Q}}^{(2)}=71.7 \mathrm{kHz}$. When the quantum dot is occupied with a single electron, the Rabi oscillations retain the same frequency but they decay sooner (decay time constant of $30 \mu \mathrm{s}$ ). When the quantum dot is occupied with two electrons, the Rabi oscillations behave exactly as for an empty quantum dot. Figure $2 \mathrm{~b}$ shows also a Rabi experiment performed at the ${ }^{115}$ In central transition. The period of the Rabi oscillations, noticeably shorter than that of ${ }^{75}$ As, reflects both the different gyromagnetic ratio and the increase in the effective coupling (a factor of five for the spin-9/2 ${ }^{115} \mathrm{In}$ ). The decay of the Rabi oscillations follows the same pattern as for ${ }^{75}$ As: the decay is the same for the empty and doubly charged states, but is more pronounced for the singly charged state.

The Rabi oscillations provide the first indication that the nuclear spin dynamics depend on the quantum dot charge. In fact, they reveal a dependence on the electron spin: there is a lone spin in the $1 e$ state but the two electrons in the $2 e$ state form a spin
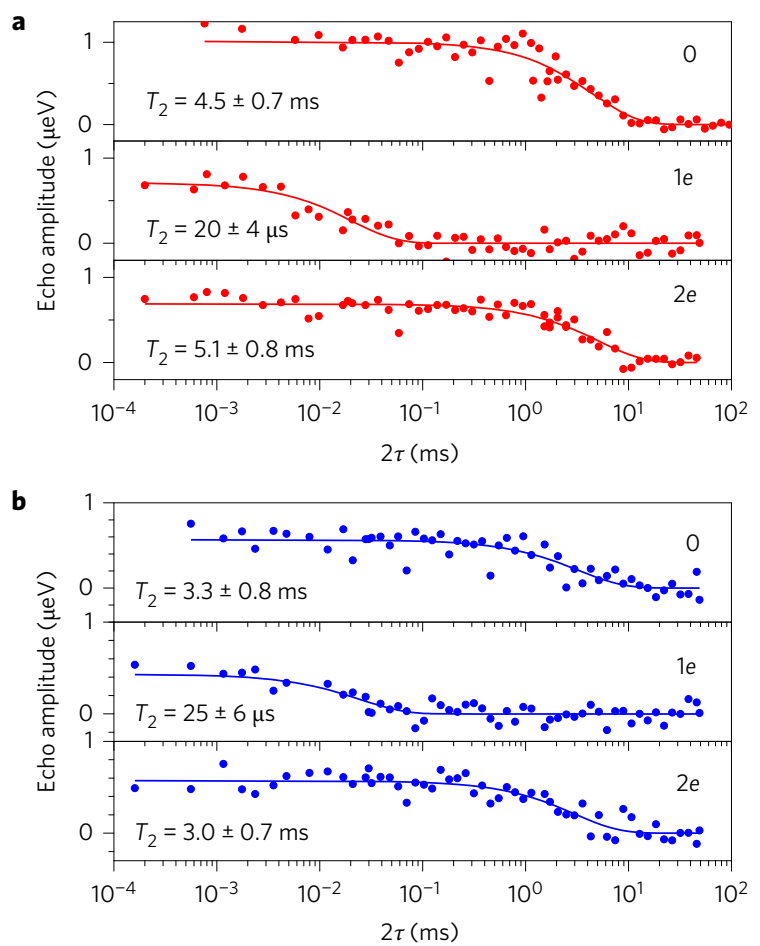

Figure 3 | Hahn echo $T_{\mathbf{2}}$ measurement. a,b, The NMR signal following a Hahn echo sequence for As (a) and In (b) for the three charge states $(0,1 e$ and $2 e)$. The Hahn echo consists of the standard $\pi / 2-\tau-\pi-\tau-\pi / 2$ sequence. The echo amplitude is plotted against the total delay $2 \tau$. We use single exponential fits $\left(\exp \left(-2 \tau / T_{2}\right)\right)$ to determine the coherence times. $T_{2}$ for the singly charged dot is more than a factor of 100 lower than for the empty or doubly charged quantum dot.

singlet. However, the faster decay of the Rabi oscillations in the presence of a single electron could signify a decreased nuclear spin coherence or an increased ensemble broadening (through the Knight field, for instance). To distinguish between these two cases, we measure the coherence associated with the nuclear central transition. The Hahn echo is perfect for this as it removes the dependence on the inhomogeneity in the quadrupole shift. Figure 3 presents the Hahn echo amplitude as a function of the echo delay for both ${ }^{75}$ As and ${ }^{115} \mathrm{In}$, in each case for three charge states. The Hahn echo for the $1 e$ state was recorded at the centre of the single-electron charging plateau. For both ${ }^{75}$ As and ${ }^{115} \mathrm{In}$, a very pronounced dependence on spin is revealed: the Hahn echo decay time $\left(T_{2}\right)$ decreases by more than two orders of magnitude in the presence of a lone electron spin.

\section{Nuclear and electron spin decoherence}

For an empty quantum dot $T_{2}$ is a few milliseconds for both ${ }^{75} \mathrm{As}$ and ${ }^{115} \mathrm{In}$, in agreement with previous experiments ${ }^{16}$. The general timescale points to decoherence via a dipole-dipole interaction. For a singly occupied quantum dot, however, $T_{2}$ falls to just $\sim 20 \mu \mathrm{s}$, a timescale that is far too short for a dipole-dipole interaction, and an extra decoherence mechanism clearly applies. Figure 4 shows $T_{2}$ versus bias, marking the extent of the $1 e$ charging plateau. Far from the charging bias, $T_{2}$ is independent of the bias for the 0 state, falling monotonically as the charging plateau is crossed. $T_{2}$ reaches a minimum at the centre of the $1 e$ plateau. In fact $T_{2}$ is symmetric about the centre of the $1 e$ charging plateau, recovering completely in the $2 e$ plateau. This is a striking result: the nuclear spins are least coherent in the centre of the $1 e$ plateau when the electronic degrees of freedom (charge, electron spin, exciton) are most coherent. 
a

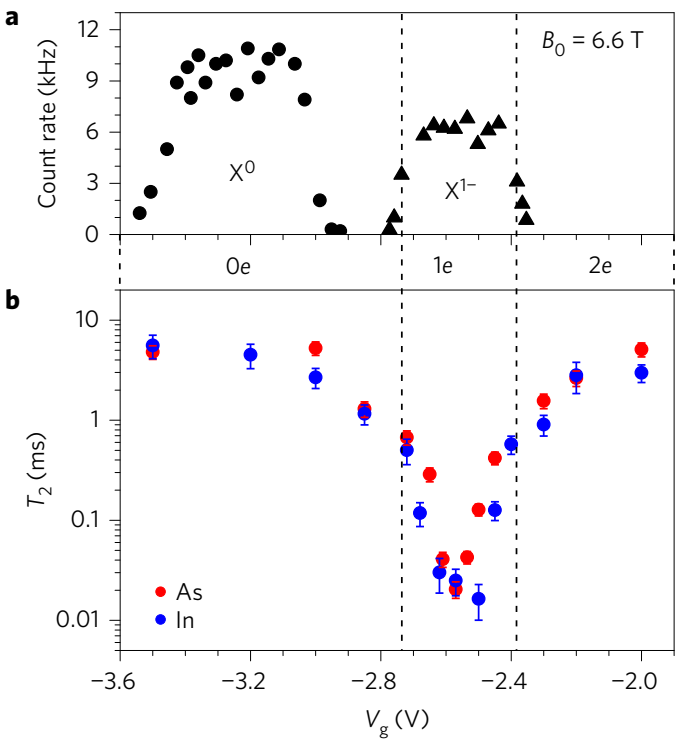

Figure 4 | Nuclear spin coherence time as a function $\boldsymbol{V}_{\mathbf{g}}$. a, Peak resonance fluorescence intensity for both neutral $\left(X^{0}\right)$ and charged $\left(X^{1-}\right)$ excitons versus the bias for a constant laser intensity. Dashed lines correspond to an $\mathrm{X}^{1-}$ emission intensity drop of $50 \%$ and indicate the boundaries of the charging plateau. At the boundary on the left the 0 and $1 e$ states are degenerate and are thus occupied 50:50; equivalently, at the boundary on the right, the $1 e$ and $2 e$ states are degenerate. $\mathbf{b}, T_{2}$ versus $V_{\mathrm{g}}$. The error bars correspond to the uncertainty in the fit to the Hahn echo measurements.

In the $1 e$ plateau, the electron spin relaxation time $T_{1}^{e}$ and the nuclear spin relaxation time $T_{1}$ follow exactly the opposite dependence on bias to the nuclear spin $T_{2} . T_{1}^{e}$ is very small close to the edges of the $1 e$ plateau edge on account of co-tunnelling (the quantum dot electron spin relaxes rapidly by swapping its spin with an electron in the Fermi sea) ${ }^{29-31}$. In the centre of the le plateau, co-tunnelling is suppressed at low temperature by the gap between the quantum dot ground state and the Fermi energy of the Fermi sea such that $T_{1}^{e}$ increases (by about four orders of magnitude with respect to the plateau edge ${ }^{29}$ ). The electron $T_{1}$ process determines the nuclear spin $T_{1}$ process: the nuclear spin leaks into the Fermi sea ${ }^{32}$. This anticorrelation between electron spin relaxation and the nuclear spin coherence is particularly pronounced at the plateau edge. Here electron spins relax extremely rapidly (revealed by an increase in the optical resonance fluorescence linewidth) and the nuclear spin polarization decays relatively quickly (see Supplementary Information). Nevertheless, this rapid electron relaxation has a relatively benign effect on the nuclear spin decoherence. The recovery of the nuclear spin $T_{2}$ in the $2 e$ state is also completely consistent with this link between the nuclear spin coherence and the electron spin: in the $2 e$ state, the two electrons form a singlet with zero total spin.

The experiments demonstrate that slow electron spin relaxation promotes nuclear spin decoherence. This points to a nuclear spin-nuclear spin interaction facilitated by a common interaction with an electron spin. Qualitatively, this interaction accounts for the experimental results. First, although this electron-mediated nuclear spin-nuclear spin interaction arises only in the secondorder hyperfine effects, it provides a means for many nuclei of a particular isotope in the quantum dot to couple together such that it has significant consequences. Second, the interaction is turned off in the $2 e$ ground state, a singlet, accounting for the recovery of the nuclear spin coherence in this regime. Third, electron spin relaxation via co-tunnelling is fast relative to the nuclear spin dynamics away from the centre of the plateau such that the nuclear spin ensemble interacts with a time-averaged electron spin $\left\langle S_{z}\right\rangle$. At the edges of the 1e plateau, $\left\langle S_{z}\right\rangle$ is small, suppressing the electron-mediated nuclear spin-nuclear spin interaction: this accounts for the anticorrelation between nuclear spin $T_{2}$ and electron spin $T_{1}^{e}$.

Nuclear spin decoherence in the presence of a single electron We seek a quantitative explanation of the nuclear spin $T_{2}$ in the centre of the plateau-that is, in the presence of a single electron spin prepared in either the up or down state. At the centre of the plateau, the central transitions of a particular isotope represent a closed system (that is, coupled quasi-spin-1/2 spins) as the average quadrupole splittings $(\sim 2 \mathrm{MHz}$; ref. 20$)$ are larger than the co-tunnelling rate $(\sim 0.1 \mathrm{MHz})$. Provided that the electron Zeeman energy is larger than the averaged hyperfine coupling, the electron-mediated nuclear spin-nuclear spin interaction results in a Hamiltonian ${ }^{9}$

$$
V=\frac{1}{8 \omega} \sum_{j \neq l} A_{j} A_{l}\left[\left(\frac{1}{2}+S_{z}\right)\left(I_{j}^{-} I_{l}^{+}+I_{l}^{-} I_{j}^{+}\right)-\left(\frac{1}{2}-S_{z}\right)\left(I_{j}^{+} I_{l}^{-}+I_{l}^{+} I_{j}^{-}\right)\right]
$$

where $\omega$ is the electron Zeeman energy (in the total magnetic field, external magnetic field plus Overhauser field), $A_{j}$ is the hyperfine coupling constant of the jth nuclear spin, $S_{z}$ is the $z$ component of the electron spin operator and $I_{j}$ is the nuclear spin operator of the $j$ th nuclear spin. The terms $I_{j}^{-} I_{l}^{+}+I_{l}^{-} I_{j}^{+}$and $I_{j}^{+} I_{l}^{-}+I_{l}^{+} I_{j}^{-}$represent nuclear spin-nuclear spin flip-flop processes and these terms lead to nuclear spin decoherence. The dynamics of the transverse components of a single nuclear spin are described in the presence of a coupling to all the others using a master equation. We treat $V$ as a perturbation and retain terms to second order in $V$. We then calculate the ensemble decoherence rate (see Supplementary Information). Despite the complexity of the problem, an analytical result for the ensemble decoherence rate $\hat{\Gamma}$ for ${ }^{75} \mathrm{As}$ can be derived

$$
\hat{\Gamma}_{\mathrm{As}}=\frac{2 \sqrt{2} A_{\mathrm{As}}^{3}}{9 \hbar \omega^{2} N}
$$

where $A_{\mathrm{As}}$ is the hyperfine constant of ${ }^{75} \mathrm{As}$ in GaAs. The total number of nuclear spins in the quantum dot $N$ is known from other experiments, $N=(8.5 \pm 0.9) \times 10^{4}$ (ref. 23). The standard value of the hyperfine constant used in the literature ${ }^{33,34}$ is $A_{\mathrm{As}}=86 \pm 10 \mu \mathrm{eV}$. The Zeeman energy under these conditions, $\omega=246 \pm 30 \mu \mathrm{eV}$, is measured in situ and we note that the condition $A<\omega$ is met in the experiment. The final theoretical result is that $1 / \hat{\Gamma}_{\mathrm{As}}=17 \pm 5 \mu \mathrm{s}$. The error specified represents a random error arising from the uncertainties in the input parameters. The experimental result for ${ }^{75} \mathrm{As}$ in the centre of the plateau is $T_{2}=20 \pm 4 \mu \mathrm{s}$ (Fig. 4): the experimental and theoretical results agree within the error bars. Away from the centre of the plateau, the co-tunnelling rate increases above $v_{Q}^{(1)}$ eventually becoming larger than the total spread in $v_{Z}(50 \mathrm{MHz}$ in this experiment) such that all of the transitions of all of the nuclei can in principle be coupled together via the common interaction with the electron; in practice, the co-tunnelling rapidly reduces $\left\langle S_{z}\right\rangle$, shutting off the interaction. A complete theory in the co-tunnelling regime is formidably complex. This comment notwithstanding, the agreement with the theoretical result at the centre of the plateau adds considerable weight to our assertion that an electron-mediated interaction is responsible for the decoherence of the nuclear spins in the presence of a single electron.

The nuclear spin coherence can be increased by applying larger magnetic fields (hard to achieve in this experiment as the DNP takes unreasonably long times) or by increasing the size of the quantum dot. We note that even for the quantum dot used here, spin rotations 
on a picosecond timescale ${ }^{35}$ can be achieved, allowing many operations to be performed before coherence is lost. Here we have shown that probing the nuclear spins in the quantum dot is a sensitive probe of the interactions turned on by the presence of a single electron. The loss of nuclear spin coherence can thus be attributed unambiguously to an electron-mediated nuclear spin-nuclear spin coupling.

\section{Methods}

Methods and any associated references are available in the online version of the paper.

Received 12 January 2016; accepted 23 May 2016; published 11 July 2016

\section{References}

1. Loss, D. \& DiVincenzo, D. P. Quantum computation with quantum dots. Phys. Rev. A 57, 120-126 (1998).

2. Chekhovich, E. A. et al. Nuclear spin effects in semiconductor quantum dots. Nature Mater. 12, 494-504 (2013).

3. Petta, J. R. et al. Coherent manipulation of coupled electron spins in semiconductor quantum dots. Science 309, 2180-2184 (2005).

4. Reilly, D. J. et al. Suppressing spin qubit dephasing by nuclear state preparation. Science 321, 817-821 (2008).

5. Shulman, M. D. et al. Suppressing qubit dephasing using real-time Hamiltonian estimation. Nature Commun. 5, 5156 (2014).

6. Bluhm, H. et al. Dephasing time of GaAs electron-spin qubits coupled to a nuclear bath exceeding $200 \mu$ s. Nature Phys. 7, 109-113 (2011).

7. Witzel, W. M. \& Sarma, S. D. Multiple-pulse coherence enhancement of solid state spin qubits. Phys. Rev. Lett. 98, 077601 (2007).

8. Cywiński, L., Witzel, W. M. \& Das Sarma, S. Electron spin dephasing due to hyperfine interactions with a nuclear spin bath. Phys. Rev. Lett. 102, $057601(2009)$

9. Klauser, D., Coish, W. A. \& Loss, D. Nuclear spin dynamics and Zeno effect in quantum dots and defect centers. Phys. Rev. B 78, 205301 (2008).

10. Khaetskii, A. V., Loss, D. \& Glazman, L. Electron spin decoherence in quantum dots due to interaction with nuclei. Phys. Rev. Lett. 88, 186802 (2002).

11. Yao, W., Liu, R.-B. \& Sham, L. J. Theory of electron spin decoherence by interacting nuclear spins in a quantum dot. Phys. Rev. B 74, 195301 (2006).

12. Hanson, R., Kouwenhoven, L. P., Petta, J. R., Tarucha, S. \& Vandersypen, L. M. K. Spins in few-electron quantum dots. Rev. Mod. Phys. 79, 1217-1265 (2007).

13. Coish, W. A., Fischer, J. \& Loss, D. Free-induction decay and envelope modulations in a narrowed nuclear spin bath. Phys. Rev. B 81, 165315 (2010).

14. Faribault, A. \& Schuricht, D. Integrability-based analysis of the hyperfineinteraction-induced decoherence in quantum dots. Phys. Rev. Lett. 110, 040405 (2013)

15. Kondo, Y. et al. Multipulse operation and optical detection of nuclear spin coherence in a GaAs/AlGaAs quantum well. Phys. Rev. Lett. 101, 207601 (2008).

16. Chekhovich, E. A., Hopkinson, M., Skolnick, M. S. \& Tartakovskii, A. I. Suppression of nuclear spin bath fluctuations in self-assembled quantum dots induced by inhomogeneous strain. Nature Commun. 6, 6348 (2015).

17. Reilly, D. J. et al. Exchange control of nuclear spin diffusion in a double quantum dot. Phys. Rev. Lett. 104, 236802 (2010).

18. Warburton, R. J. et al. Optical emission from a charge-tunable quantum ring. Nature 405, 926-929 (2000).

19. Urbaszek, B. et al. Nuclear spin physics in quantum dots: an optical investigation. Rev. Mod. Phys. 85, 79-133 (2013).
20. Munsch, M. et al. Manipulation of the nuclear spin ensemble in a quantum dot with chirped magnetic resonance pulses. Nature Nanotech. 9, 671-675 (2014).

21. Chekhovich, E. A. et al. Structural analysis of strained quantum dots using nuclear magnetic resonance. Nature Nanotech. 7, 646-650 (2012).

22. Bulutay, C., Chekhovich, E. A. \& Tartakovskii, A. I. Nuclear magnetic resonance inverse spectra of InGaAs quantum dots: atomistic level structural information. Phys. Rev. B 90, 205425 (2014)

23. Kloeffel, C. et al. Controlling the interaction of electron and nuclear spins in a tunnel-coupled quantum dot. Phys. Rev. Lett. 106, 046802 (2011).

24. Warburton, R. J. Single spins in self-assembled quantum dots. Nature Mater. 12, 483-493 (2013).

25. Kuhlmann, A. V. et al. Charge noise and spin noise in a semiconductor quantum device. Nature Phys. 9, 570-575 (2013).

26. Kuhlmann, A. V. et al. A dark-field microscope for background-free detection of resonance fluorescence from single semiconductor quantum dots operating in a set-and-forget mode. Rev. Sci. Instr. 84, 073905 (2013).

27. Latta, C. et al. Confluence of resonant laser excitation and bidirectional quantum-dot nuclear-spin polarization. Nature Phys. 5, 758-763 (2009)

28. Van Veenendaal, E., Meier, B. \& Kentgens, A. Frequency stepped adiabatic passage excitation of half-integer quadrupolar spin systems. Mol. Phys. 93, 195-213 (1998).

29. Smith, J. M. et al. Voltage control of the spin dynamics of an exciton in a semiconductor quantum dot. Phys. Rev. Lett. 94, 197402 (2005).

30. Dreiser, J. et al. Optical investigations of quantum dot spin dynamics as a function of external electric and magnetic fields. Phys. Rev. B 77, 075317 (2008).

31. Kroner, M. et al. Resonant two-color high-resolution spectroscopy of a negatively charged exciton in a self-assembled quantum dot. Phys. Rev. B 78, 075429 (2008).

32. Maletinsky, P., Badolato, A. \& Imamoglu, A. Dynamics of quantum dot nuclear spin polarization controlled by a single electron. Phys. Rev. Lett. 99, 056804 (2007)

33. Paget, D., Lampel, G., Sapoval, B. \& Safarov, V. I. Low field electron-nuclear spin coupling in gallium arsenide under optical pumping conditions. Phys. Rev. B 15 5780-5796 (1977)

34. Coish, W. A. \& Baugh, J. Nuclear spins in nanostructures. Phys. Status Soldi B 246, 2203-2215 (2009).

35. Press, D. et al. Ultrafast optical spin echo in a single quantum dot. Nature Photon. 4, 367-370 (2010)

\section{Acknowledgements}

R.J.W., M.P. and D.L. acknowledge support from NCCR QSIT; R.J.W. and D.L. from EU ITN S ${ }^{3}$ NANO; R.J.W. from SNF project 200020_156637; M.P. from the SNI; and A.L. and A.D.W. from Mercur Pr-2013-0001 and BMBF-Q.com-H 16KIS0109.

\section{Author contributions}

G.W. and M.M. carried out the experiments, the data analysis and the theoretical modeling under the guidance of M.P. and R.J.W.; F.M. conducted the theoretical analysis under the guidance of D.L.; A.V.K. provided expertise in resonance fluorescence on single quantum dots; A.L. and A.D.W. carried out the molecular beam epitaxy. G.W., M.M., F.M. and R.J.W. took the lead in writing the paper and the Supplementary Information. R.J.W. managed the project.

\section{Additional information}

Supplementary information is available in the online version of the paper. Reprints and permissions information is available online at www.nature.com/reprints. Correspondence and requests for materials should be addressed to M.M.

\section{Competing financial interests}

The authors declare no competing financial interests. 


\section{Methods}

The InGaAs quantum dots are embedded in a vertical tunnelling structure consisting of a Shottky diode, with the quantum dots in tunnel contact with an $\mathrm{n}$-doped layer via a $25 \mathrm{~nm}$ thick intrinsic GaAs layer. Resonance fluorescence from the quantum dot is excited with a coherent continuous wave laser and collected with very high signal to background ratio with a polarization-based dark-field technique. On the neutral exciton, $\mathrm{X}^{0}$, the linewidth is just $1.5 \mu \mathrm{eV}$ at zero magnetic field, close to the transform limit, and at resonance the count rates on a silicon avalanche photodiode are $100 \mathrm{kHz}$. In an applied magnetic field the 'dragging' of the resonance under resonant excitation is used to polarize the nuclear spins to achieve $\mathrm{DNP}^{27}$. An $\mathrm{Au}$ microwire is positioned on an insulating layer directly above the quantum dot, with a hole for optical access, Fig. 1d. We achieve a peak-to-peak radiofrequency magnetic field of $5 \mathrm{mT}$. The nuclear spins are prepared (DNP followed by population swapping) on an empty quantum dot. The preparation pulses use a chirp of $10 \mathrm{GHz} \mathrm{s}^{-1}$ (which ensures adiabaticity at the first quantum transition ${ }^{20}$ ). The laser is then turned off and a bias voltage $V_{\mathrm{g}}$ is chosen for the NMR manipulation. Following the NMR protocol (Rabi pulse or Hahn echo sequence), the bias is reset to its initial value and the nuclear spin polarization is read out by turning the laser back on. The change in the onset of the optical signal on tuning the laser from large negative values constitutes the NMR signal ${ }^{20}$. The theory uses the NakajimaZwanzig master equation and the Born-Markov approximation. Technical details are provided in the Supplementary Information. 